\title{
Contributions of the Anterolateral Complex and the Anterolateral Ligament to Rotatory Knee Stability in the Setting of ACL Injury: A Roundtable Discussion
}

\author{
V Musahl, A Getgood, P Neyret, S Claes, JM Burnham, C Batailler, B Sonnery-Cottet, A Williams, A \\ Amis, S zaffagnini, J Karlsson.
}

\section{Introduction:}

Persistent rotatory knee laxity is increasingly recognized as a common finding after anterior cruciate ligament (ACL) reconstruction. While the reasons behind rotatory knee laxity are multifactorial[11,50,42,39,31,26], the impact of the anterolateral knee structures (including the anterolateral ligament, or ALL, and the anterolateral complex, or ALC) is significant[45,43]. As such, substantial focus has been directed toward better understanding these structures, including their anatomy, biomechanics, in-vivo function, injury patterns, and the ideal procedures with which to address any rotatory knee laxity that results from damage to these structures. In fact, the recent renewed awareness of the anterolateral knee structures has resulted in a proliferation of studies on this topic [47] over the last several years.

Despite the resurgence of interest in these structures, the complexity of lateral knee anatomy, varying dissection techniques, differing specimen preparation methods, inconsistent sectioning techniques in biomechanical studies, and confusing terminology have led to discrepancies in published studies on the topic. Furthermore, since the classic descriptions of anterolateral knee anatomy by Kaplan, Hughston et al., Terry et al., and Mueller in the 1950s-1980's[44,23,13,16], anatomical and functional descriptions have varied widely[27,19]. This has led many authors to call for unity and consistency in future publications and descriptions of the ALL and the ALC.[27,19] Additionally, outcomes research exploring the indications for and long-term effects of lateral extra-articular procedures is in a state of relative infancy compared to isolated primary intra-articular ACL reconstruction. As such, we have assembled a panel of expert surgeons and scientists to discuss the roles of the anterolateral structures in rotatory knee laxity, the healing potential of these structures, the most appropriate procedures to address rotatory knee laxity, and the indications for these procedures.

In this round table discussion, KSSTA Editor-in-Chief Professor Jón Karlsson poses a variety of relevant and timely questions. Andrew Amis (United Kingdom), Steven Claes (Belgium), Alan Getgood (Canada), Volker Musahl (United States), Philippe Neyret (France), Bertrand Sonnery-Cottet (France), Andy Williams (United Kingdom), and Stefano Zaffagnini (Italy) provide answers based on their personal experiences, scientific study, and interpretations of the literature. We hope this healthy scientific debate will constitute a substantial step toward refining the classification, terminology, and understanding of anterolateral knee injuries in ACL-deficient knees.

Karlsson: The anterolateral complex (ALC) has been described as including the iliotibial band (ITB), the Kaplan fibers, the capsulo-osseous layer of the ITB, and the anterolateral capsule (Figure 1). Some authors would also include the anterolateral ligament (ALL) when discussing this complex. Which of these structures do you consider to be critical to rotatory knee stability?

Amis: I believe that all of these structures make some contribution to knee stability, particularly what is known as 'anterolateral rotatory instability (ALRI).' However, that pattern of instability and its prevention also depends on the integrity of the ACL. Recent robot work by Kittl et al. measured the contributions of some of the structures listed, and they found 
that the ITB and its attachments to the femur and anterolateral tibia were the most important. The ACL had a significant role, but only in the extended knee. The ALL and associated capsule had very little effect, they were dominated by the overlying fibres of the ITB[18].

Getgood: Similar to the posterolateral corner of the knee, I believe that the anterolateral complex is a series of structures, all of which play a role in controlling anterolateral rotatory laxity. Biomechanical studies have shown that the ITB, along with its Kaplan fibre attachment of the deep capsulo-osseous layer, play the most important role in controlling internal rotation throughout the flexion range[18]. Most recently the anterolateral ligament has been shown to also provide secondary rotatory stability, although mostly in greater flexion angles over 35 degrees $[51,30,33]$. This would therefore question its role in controlling the pivot shift, a phenomenon that is observed at angles of 0 to 30 degrees of flexion. I also believe the lateral meniscus should be thought of as part of the anterolateral complex. Musahl et al. showed that lateral meniscal loss had a significant role in the manifestation of the pivot shift[24], with our own studies most recently suggesting that it plays a role in controlling internal rotation in low flexion angles, whilst also having an intimate relationship with the ALL attachment at the tibia (submitted for publication). These structures therefore all work together to provide anterolateral rotatory stability, with recent imaging studies showing a high prevalence of injury to these structures in combination with ACL rupture[48].

Neyret: We believe there is no evidence on the respective role of the different structures. Undoubtedly the ITB and the Kaplan fibers play a role. One can hypothesize the anterolateral ligament (the superficial one and the deepest structures we re-described with Vincent et al.)[49] play a role.

Sonnery-Cottet: As demonstrated by Claes et al, it is clear that the nomenclature used to describe the components of the ALC has been a cause for confusion. It should therefore be highlighted that the deep capsulo-osseous layer of the ITB and the ALL are seemingly synonymous, particularly as described by Terry et al. in 1986[44]. From a biomechanical point of view, Nitri et al, Rasmussen et al. and our group demonstrated that the ITB, the ALL and the ACL all play critical roles in anterolateral rotatory instability[29,33]. However, it is important to note that significant rotational instability requires a combined injury to the ACL and either the ITB or the ALL. The latter appears to occur frequently, with Van Dyck et al demonstrating concomitant ALL injury on MRI in approximately 50\% of patients with an ACL injury[48] and Ferretti et al demonstrating an incidence of $90 \%$ after a systematic anterolateral surgical exploration[10].

Williams: The ITB through to Kaplan fibres. The capsule and ALL are not important.

Claes: The ALL is absolutely critical in providing an adequate restraint to excessive internal rotation in physiological loading conditions in the ACL-deficient knee, as is demonstrated by the occurrence of a Segond fracture in up to $10 \%$ of the clinical ACL injuries. The ITB and its described subdivisions might have a role in controlling rotation in the laboratory setting, but clinical injuries have only been reported in extremely rare cases. Given the overwhelming evidence in recent literature for a definitive role of the ALL in controlling knee rotation, it seems no longer correct to describe this ligament in rather confusing terminology as Kaplan 
fibers, capsulo-osseous layer or anterolateral capsule (ALC).

Musahl: The anterolateral complex as a whole is important for rotatory knee stability. Kittl and Amis et al. showed that the ITB and its deep fibers are the most important[18]. We were able to show the same in our laboratory[32]. Using a robotic manipulator, we applied a combined internal rotation and valgus load to cadaveric knees. We were able to confirm that the ACL is the primary load bearing structure near full extension. We showed that the LCL and ALC are load sharing in higher knee flexion angles while the "ALL" carried a negligible force[1].

Zaffagnini: All of the above. During different experimental studies it was possible to identify the ALL complex. Nevertheless, it is still under discussion if such identification is more correlated with the performed dissection or with the structure itself. On my personal opinion both of the aspects are relevant.

This lack of agreement is confirmed by the literature during the last years. In particular, Runer et al. confirmed the presence of the ALL in the $45.5 \%$ of the analyzed knee joints[35]. Musahl et al. found a discrete capsular thickening of 2-4 mm on MRI in adult human cadaveric specimens[26]. Conversely the works of both Kennedy et al. and Vincent et al. confirmed the presence of the ALL in the 100\% of the analyzed joints[17,49]. Concerning the laxity control, Bonanzinga et al. in an in-vitro study underlined how the ALL plays a significant role in controlling static internal rotation and acceleration during a pivot shift test[4]. Also Rasmussen et al. and Early et al. confirmed that ALL is an important lateral knee structure for rotatory and translational stability[33,9].

Karlsson: Various descriptions have been provided in the literature regarding the presence, origin, and insertion of the ALL. Do you consider this ligament to be a discrete structure, and if so, what do you consider to be the origin and insertion of the ALL?

Getgood: Our anatomic study published in KSSTA found the ALL to be present in 19 of 19 fresh frozen cadavers, although the anatomy in our specimens was found to be somewhat variable[5]. Histologically we found the ALL to be a ligamentous structure within the anterolateral complex of the knee. It is not discrete like the FCL; however, it does exhibit histological characteristics that would make it ligamentous in nature and therefore differentiates it from the surrounding capsule. This can be likened to the glenohumeral ligaments of the shoulder, or the ligaments of the elbow - dense condensations of connective tissue that provide static joint stability. The most consistent finding within our study was confirmed by Kennedy et al.[17] who documented the femoral origin to be $4.7 \mathrm{~mm}$ posterior and proximal to the lateral epicondyle with the tibial insertion midway between the anterior border of the fibular head and Gerdy's tubercle. These landmarks were further demonstrated in a radiographic study by Rezansoff et al.[34]

Neyret: There are various descriptions of the ALL according to the method of dissection. From superficial to deep layers we can dissect a thin structure superficial to the LCL. This structure is very discrete. If we consider the structure we've described previously with 
Vincent et al. and we've called the ALL, this structure is as big as the popliteus tendon and shares femoral insertion with the LCL[49]. We also perfectly see this structure with special MRI sequences.

Claes: The ALL is definitely a distinct ligamentous structure and its existence on the anterolateral aspect of the human knee is already confirmed in more than 100 peer-reviewed papers until now. Although the initial descriptions might have stirred some confusion, most authors now agree that its origin lies posterior and proximal of the lateral femoral epicondyle. The ALL inserts on the "Segond locus", at the anterolateral aspect of the proximal tibia, halfway in between the center of both Gerdy's tubercle and the fibular head.

Williams: The structure exists and has a clear distal attachment - 1/2 way between Gerdy's tubercle and the LCL attachment to the fibula head. The proximal attachment to less clear as there is a blending with capsule and periosteum. However, I support the concept of the femoral attachment being about $10 \mathrm{~mm}$ proximal and $5 \mathrm{~mm}$ posterior to the LCL attachment to the femur.

Zaffagnini: The origin of the capsular thickening structures that can be considered as ALL start from behind the epicondyle (near Kaplan fibers) and then they extend on the lateral portion of the capsule right up to the Gerdy's tubercle.

Amis: Yes, we have found the ALL reliably in our dissections, but it is a rather insubstantial structure and is easily missed. Its femoral attachment is approximately $8 \mathrm{~mm}$ proximal and 4 $\mathrm{mm}$ posterior to the lateral epicondyle. The ALL then passes superficial to the lateral (fibular) collateral ligament (LCL) and attaches to the tibia mid-way between the head of the fibula and Gerdy's tubercle, approximately $10 \mathrm{~mm}$ below the joint line.

Sonnery-Cottet: The ALL is clearly a discrete structure. This has now been demonstrated in cadaveric, clinical and imaging studies. I would recommend the surgical dissection presented by Daggett et al[8]. In this dissection, one can clearly identify the ALL as a discrete structure and see that its origin is just proximal and posterior to the lateral epicondyle. It has one insertion on the periphery of the lateral meniscus and a broad insertion on the anterolateral tibia, between Gerdy's tubercle and fibular head. A bony avulsion of this structure results in the Segond fracture, dissection of which clearly identifies the ALL as being responsible for its pathogenesis. Both the ALL and Segond fracture dissections are available on Vumedi and are entitled "The Anterolateral Ligament Exists, Now What? Part 1 of 2."

Musahl: There is the capsulo-osseous layer of the ITB, which is robust and consistently present (Herbst et al. KSSTA - submitted). There is also the mid third capsular ligament as described by Terry and Hughston[14,13,44]. The mid third capsular ligament is less consistently present and is embedded within the anterolateral capsule and is not a true ligament. A ligament is defined as collagen fibers aligned along its long axis and mechanical properties, i.e. strain predominantly in line with its tensile strength[28]. However, in our biomechanical studies, the anterolateral capsule behaves like a sheet of tissue rather than a true ligament[12]. 
Karlsson: Is it possible that ALC or ALL injuries heal on their own, similar to the MCL?

Neyret: We believe the ALL can heal. Another question is: can the ALL be stretched over the time?

Claes: Absolutely. Although the occurrence of ALL injuries has been reported in $32-90 \%$ of the ACL-injured knees on both ultrasound and magnetic resonance imaging, only a subset of these patients need actual ALL treatment. The proper delineation of this treatment group will be an important study topic in the coming years. In our own practice, growing knowledge on the ALL has driven us to treat ACL injuries more acutely in order to "brace" the injured ALL and facilitate its natural healing.

Amis: I do not know of any real evidence for this, but variability of tissue healing responses may account for part of the spectrum of residual laxity reported at follow-up. It is likely that healing in-situ would be aided by surgery or other means to control the laxity during the healing phase, including ACL reconstruction, as is well-established at the medial side of the knee.

Musahl: Most capsular injuries are shown to have good healing potential. The healing potential is likely improved if rare bony avulsions, such as Segond fracture occur. Disruptions of larger soft tissue structures such as the ITB itself usually occur as part of a muliligament injury or knee dislocation. Repair/reconstruction of these injuries is not evidence based but rather decided on a case-by-case basis; most commonly they are treated in acute situations by primary repair.

Sonnery-Cottet: It is possible that some of these lesions heal on their own like MCL tears; on the other hand, it is also possible that ACL or ALL tears will demonstrate poor healing potential like LCL tears. There is currently insufficient evidence to answer this question satisfactorily and further study is required.

Karlsson: Do all ALC and ALL injuries need to be repaired or reconstructed? Is repair or reconstruction better? Does it matter if it is a soft tissue injury as compared to a bony avulsion such as a Segond fracture?

Claes: An injured ALL should be treated if the ACL-deficient knee demonstrates a high degree of rotational instability (IKDC grade II and III), especially when you deal with a pivoting athlete in a subacute or chronic case. I certainly agree that more information is needed about the efficacy of different surgical ALL reconstruction or repair techniques, but as we've learned from ACL double bundle reconstructions, only long-term clinical outcome studies will be able to demonstrate superiority of a certain technique. In acute cases there is a role for primary ALL repair as well as in Segond fractures.

Getgood: My own personal opinion is that if the ACL is reconstructed acutely, this may prevent the development of gross anterolateral rotatory laxity. If left to the chronic stages, 
when we see much greater degrees of rotatory laxity, then an ALC reconstruction may be appropriate, particularly for young patients who have high grade laxity and those returning to pivoting sport, who are therefore at high risk of re-injury. In terms of Segond fracture repair, this intuitively makes sense. Anatomic studies have shown that both the ALL and the ITB attach to the area of the Segond avulsion. However, as seen in the ACL tibial avulsion, there is a degree of ligamentous strain which does not recover following avulsion repair, therefore repair may result in inferior outcomes. Clinical studies are needed to shed further light on this area.

Sonnery-Cottet: I feel that ALL injuries need to be repaired or reconstructed in patients with a high risk of graft re-rupture. For now, it is not possible to say if repair is better than reconstruction in acute cases, but reconstruction is certainly less invasive. This is because reconstruction is performed percutaneously, and therefore preserves the integrity of the ITB, which is of course an important structure for rotational control. In situations where there is a bony avulsion e.g. Segond fracture, I would do a repair. However, in our experience, the Segond fracture is an epiphenomenon. At our center, we perform over $1200 \mathrm{ACL}$ reconstruction a year and see no more than a few Segond fractures per year.

Williams: No, [all ALC and ALL injuries do not need to be reconstructed]. There is little to justification for ALL reconstruction over a tenodesis, as the latter performs much better in the lab. However, if there was, for some reason, a major soft tissue reconstruction, I can see a reason for repair but it would be rare. It doesn't really matter [whether it is a soft tissue or Segond fracture], but a large Segond fragment would be easier to address.

Zaffagnini: Incidence of macroscopic tears of the lateral capsule after ACL injury is significant, as Ferretti et al. reported a percentage over $90 \%$ in an in-vivo study including 60 patients[10]. I would say that repair could be executed in acute setting but more often the lesion is addressed when a chronic situation is present. Therefore, if the rotatory laxity related to this structure is present, reconstruction with lateral tenodensis is probably the best choice. If a bony avulsion is present in an acute setting probably re-fixation of the bony fragments with the structure attached and re-tensioned could be sufficient. In a chronic situation it is better to perform a reinforcement. Bony lesions remain difficult to be evaluated. Chylarecki C et al., in an experimental study on 20 cadaver knee joints, demonstrated using a radiological examination a positive correlation between ventral translation of the tibial head by $3 \mathrm{~mm}$ or more (compared with the non-injured side) and an ACL lesion[6].

Karlsson: In addition to the ACL and ALC, what other factors might contribute to the rotational stability of the knee?

Sonnery-Cottet: Bone morphology, meniscal tears (especially root tears), other peripheral ligament tears such as posteromedial corner injuries and joint hyper-laxity are certainly important factors. There may be others currently unknown as well. 
Neyret: In addition to the ACL and ALL, the posteromedial corner contributes to the rotatory stability of the knee (menisco tibial ligament, postero medial horn of the medial meniscus), the lateral meniscus and also the PCL.

Claes: In fact, the most important message to be taken from the renewed interest on anterolateral instability can be distilled in one single quote from Eduardo Monaco and Andrea Ferretti stating in 2012 that "we will never fully understand rotatory instability as long as we look only at the ACL"[22]. In this view, our work on the ALL has obviously widened the scope when dealing with ACL injured knees, but the ALL is surely not the only solution to rotatory instability in ACL-deficient knee. For instance, the so-called meniscotibial ligament at the posterior meniscal insertion on the tibia has been shown to play a role in knee stability, but intrinsic bony knee geometry is probably as important.

Williams: There is a role of many things - the natural geometry of the joint surfaces- perhaps the large lateral femoral impact on lesion also, the menisci, the whole capsule / collateral ligament structures. Out of the unseemly debate about the ALL, etc. one good thing has occurred: an appreciation of the periphery of the joint as being of considerable importance.

Amis: There is growing awareness of the roles of many structures around the knee which have some role in control of rotational stability, including the menisci and their attachments, and the medial side capsulo-ligamentous complex.

Musahl: Rotatory knee laxity is multifactorial. It is important to understand that the anterolateral complex, as well as the posteromedial corner both influence rotatory knee laxity, however not unless the central cruciate, i.e. the ACL is disrupted. Therefore, while not the only important structure, the ACL and the bony geometry dictate rotatory knee laxity.

Zaffagnini: In addition to the ACL and ALC superficial and deep MCL lesion could contribute to anteromedial rotational laxity and it is often difficult from the clinical examination to really identify the structure involved when anteromedial and anterolateral structures are injured. Moreover especially in chronic setting there is a plastic deformation of all the capsular structures that contribute to increase the knee rotation. Meniscal lesion and meniscal removal especially of the lateral meniscus can be responsible of an increased rotational laxity.

There are also others factors that affect the laxity of the knee joint like anatomy[26,27,31], preoperative laxity level[37], time injury-to-surgery[38], as well as combined lesion such as meniscal lesion[3].

Karlsson: Describe the ideal patient that would benefit from an extra-articular tenodesis or reconstruction procedure in addition to ACL reconstruction.

Getgood: At present there is very little high level evidence to guide decision making as to whom will benefit from [lateral extra-articular tenodesis] LET. We are presently performing a randomized clinical trial (Stability Study; Clinical Trials.gov NCT02018354) comparing 
ACL reconstruction with or without LET augmentation in individuals who are deemed at being high risk of graft failure. We have determined high-risk individuals as those under the age of 25 years old with high-grade rotatory laxity (pivot shift grade 2 or greater) and/or have generalized ligamentous laxity (or knee recurvatum greater than 10 degrees), who are returning to pivoting sport. At present we have recruited 545 patients in this multicenter study across nine centres in Canada and Europe and hope that the results will help determine who will most benefit from this procedure, if at all. Otherwise I perform LET on patients undergoing revision surgery in which there are no other laxities that need to be addressed (e.g posterolateral rotatory laxity)[46]. In the primary scenario, for those who do not consent or are ineligible to be in the RCT, I perform LET on young patients who have a pivot shift grade 3 with generalized ligamentous laxity, particularly in those who are wishing to return to pivoting sport.

Musahl: Our indications currently for extra-articular tenodesis are patients with a high-grade rotatory knee laxity (lateral compartment translation $>5 \mathrm{~mm}$; tibial acceleration $>10 \mathrm{~m} / \mathrm{sec}^{2}$ ) and patients in whom excess rotatory knee laxity cannot be addressed by meniscus repair/reconstruction, root repair, posteromedial corner repair, or osteotomy.

Sonnery-Cottet: I consider there to be 3 main reasons to perform extra-articular reconstruction in addition to ACL reconstruction. The most important is to decrease the ACL graft rupture rate. The second is to improve rates of return to play at the pre-injury level of sport, and the third is to potentially improve rotational stability. On that basis, the ideal patient would be one who is predicted to have a high risk of graft rupture. This could include young patients involved in pivoting sports. However, we should question whether restricting the surgical indication to just high risk patients is appropriate. Our forthcoming clinical series demonstrates a significant decrease in ACL graft rupture rates and improved return to sport when an ALL reconstruction is also performed. At the same time, we have not noted specific complications with a minimally invasive ALL reconstruction technique. Based on these considerations, the risk/benefit ratio appears to dramatically favour combined ACL/ALL procedures.

Neyret: In our practice, we consider extra-articular tenodesis when there is:

1. A large amount of anterior tibial translation of the lateral tibial plateau.

2. In case of revision ACL reconstruction (particularly if the primary surgery was well done).

3. This procedure can also be discussed with patients at risk of re-rupture, return to strenuous sports, or explosive jerk test.

Claes: An ACL-injured pivoting athlete with a high-grade pivot-shift (IKDC grade II-III) with typical bone edema on the distal femoral condyle and posterolateral tibial plateau, a deep lateral femoral notch sign and direct evidence for ALL injury on both MRI and ultrasound should not be withheld from proper ALL reconstruction. The vast majority of ACL revision cases with massive rotatory instability need ALL reconstruction too. In our opinion, the specific ALL reconstruction technique can be open for debate, but not these indications. 
Williams: This is not known but I do a modified Lemaire tenodesis in all juveniles (intra-op radiograph ensures fixation distal to the growth plate), those with a big pivot shift, big hyperextenders, people with loose ligaments, a strong family history or contralateral ACL rupture, and I have a lower threshold in professional sportsmen and women. I admit it remains subjective.

Amis: I am not aware of any high-level evidence to answer this with confidence, but the consensus is moving towards treatment of high-demand patients who have large rotational laxity post-injury.

Zaffagnini: The ideal patient depends on the injury pattern. To schematize:

- All patients with high rotatory laxity measured with quantitative system like KiRA device[1] or Ipad application[25] (i.e. KiRA value > 2.4)

- All patients operated after more than 1-year from injury

- Patients presenting a high tibial lateral slope

- Patients performing sport with high demand for performance

Karlsson: Which procedure do you personally prefer when performing an extra-articular tenodesis or reconstruction surgery with concomitant ACL reconstruction?

Getgood: My preferred procedure is what I call a modified Lemaire LET, as I credit David Dejour from Lyon, from whom I learnt the technique. This involves harvesting a $1 \mathrm{~cm}$ wide 8 $\mathrm{cm}$ long strip from the posterior half of the ITB, leaving it attached at Gerdy's tubercle. The free end is whip stitched and tunneled under FCL at its femoral origin. It is then attached to the metaphyseal flare of the lateral femoral condyle with a staple at 60 degrees of flexion, neutral tibial rotation.

Zaffagnini: I personally perform a single bundle plus lateral plasty (SBLP) reconstruction described by Marcacci and Zaffagnini in 1998[21]. It is a combined intra- and extra-articular arthroscopic ACL reconstruction with hamstring tendons. After drilling of the tibial tunnel, an over-the-top arrangement is performed. The tendons are then fixed with double staples in the groove created in the posterolateral aspect of the femur. The remaining part is fixed distally to Gerdy's tubercle passing under the fascia, but over the lateral collateral ligament. This technique ensures sufficient strength in the graft and permits correction of any associated instability, because of the presence of the extra-articular portion of the tendons. Moreover, Bignozzi et al. in 2009 showed, in an in-vivo study with navigation system, how in the previously described technique the addition of the extra-articular procedure may be effective in controlling tibial translation during anterior-posterior stress test[2]. Long-term results after more than 10 years confirm the good clinical and radiographic results[20].

Neyret: I personally perform a Lemaire type procedure (using $10 \mathrm{~mm}$ width of fascia lata or gracilis) and the graft is passed under the LCL and superficial to the popliteus tendon. It's attached on the Gerdy on the tibia and posterior to the LCL on the femur at the top of the angle formed by the lateral gastrocnemius and the LCL. 
Claes: We most often perform minimally invasive anatomic ALL reconstruction using a gracilis autograft, although in the ACL revision cases we will often use a modified ITB tenodesis technique.

Sonnery-Cottet: We have described minimally invasive anatomical ALL reconstruction using the gracilis graft, sutured to a tripled semitendinosus ACL graft. The tunnels are drilled outside-in and the same femoral tunnel is used for both ACL and ALL reconstruction. The ALL reconstruction can also be performed separately. However, the main message to emphasize is that this procedure is performed percutaneously to avoid iatrogenic injury to the ITB.

Williams: The lab testing showed ALL does not perform well (for us) and only works at all with over tensioning graft[36]. MacIntosh and modified Lemaire perform well if taken deep to LCL, and only over-constrain if over-tensioned and with the foot fixed in external rotation. So for most I use a modified Lemaire $(1 \mathrm{~cm}$ wide strip of ITB attached to Gerdy, taken deep to LCL and fixed to lateral femur proximal and posterior to LCL attachment to femur with a suture anchor and then fold remaining $2 \mathrm{~cm}$ of graft back on itself over LCL and suturre it to itself, and close ITB defect. In some revisions if the ITB looks thin I do a MacIntosh: $1 \mathrm{~cm}$ wide strip ITB taken deep to LCL and fixed to lateral femoral metaphysis with a soft tissue staple.

Musahl: I will perform a modified Lemaire if using quadriceps tendon (preferred) as my primary ACL graft. Sometimes in revision ACL surgery, allograft is helpful for filling an enlarged tibial tunnel with calcaneus bone block. I prefer using a modified Marcacci technique when using allograft, e.g. an Achilles tendon bone block is in fixed in the tibial tunnel, the tendon is tubularized $(20 \mathrm{~cm})$ and fixed over-the-top with a staple. The tendon is passed deep to the ITB and fixed posterior to Gerdy's tubercle with a second staple[21].

Karlsson: Approximately what percentage of your ACL reconstructions also receives some type of extra-articular tenodesis or reconstruction procedure?

Getgood: Due to the current randomized controlled trial that we are performing, the number of LET procedures in the primary ACL population that I am performing is somewhat skewed. Prior to the study I would have performed an LET in less than 5\% of primary ACL reconstructions, with approximately $80 \%$ of revisions having one.

Neyret: It represents approximately $25 \%$ of my practice in primary ACL reconstruction and $50 \%$ in revision surgery.

Sonnery-Cottet: $46 \%$ in 2015, 60\% in 2016. Our indications include patients with a high risk of graft failure: Revision procedures, young age, pivoting sports, side to side laxity $>7 \mathrm{~mm}$, deep lateral femoral notch sign on X-rays, Pivot shift grade 2 or 3, and Segond fracture. Our question at the present time is to know whether we should extend our indications to all patients because we have significantly improved our clinical results - in particular the graft failure rate.

Musahl: In 5-10\% of primary ACL reconstructions. In 10-20\% of revision ACL 
reconstructions (note: the majority of revisions receive additional root repair, meniscus transplant, or osteotomy to restore rotatory knee stability).

Claes: In up to 25-35\% of our ACL reconstruction cases and almost all revision ACL reconstructions, concomitant ALL reconstruction is considered necessary.

Williams: $100 \%$ revisions, and 50\% primaries (note 50\% of all my ACLRs are in professional athletes).

Zaffagnini: Around 80\%.

Karlsson: In your experience, what have the outcomes been after performing extra-articular tenodesis or reconstruction procedures with ACL reconstruction?

Getgood: Our experience to date has been very encouraging. We have not seen significant down sides of performing an LET in the primary ACL reconstruction scenario. Subjective clinical assessment is very encouraging with many patients having no residual pivot shift. Strength testing and functional testing show statistical reduction of quads strength in the LET group at 6 months, normalizing by 12 months. However, the data does show that these deficits are not clinically relevant. It remains to be seen if the addition of the LET does actually reduce graft failure, or reduce persistent rotatory laxity. Stay tuned for one year results to be presented in 2017 !

Claes: In our experience, the results of combined ACL + ALL reconstructions have been excellent so far. In the short term we have seen a decrease in ACL graft re-rupture rates without causing extra morbidity[41]. Patients undergo the same post-operative regimen and rehabilitation protocol than isolated ACL reconstructions.

Neyret: In 2002 we did, with my team, a prospective study "isolated versus "combined" with extraarticular tenodesis ACL reconstruction". This study was published in the Journées Lyonnaises de Chirurgie du Genou[15]. This global IKDC evaluation was very similar in the two groups but the control of the Jerk test, as well as the control of the anterior translation in the lateral compartment, were better in the combined groups. But the flexion was $10^{\circ}$ better in the isolated ACL reconstruction group.

Zaffagnini: Highly successful.

Sonnery-Cottet: In our experience, in a high risk population (16-30 years old, practicing sport pivoting), patients with a combined ALL and ACL reconstruction have a lower graft rupture risk; 2.5 less than with isolated B-PT-B and 3.1 times less than isolated hamstring graft. We also found a higher rate of return to the pre-injury level of sport, and less pivot shift than patients that undergo ACL reconstruction alone.

Karlsson: What are the potential downsides to performing extra-articular tenodesis or reconstruction 
Getgood: A number of studies have shown an increased risk of infection with the addition of the LET[40]. We have not seen this within our study; however, the sample size is too small to study such an outcome variable. Complications that we routinely counsel our patients on are the presence of an extra scar with associated swelling and bruising, the risk of haematoma (damage to the superior lateral geniculate artery) and hardware irritation from the staple.

Neyret: The potential downsides are mainly to over constrain the rotation of the lateral compartment. It could limit the external rotation. It's a kind of stiffness, not in flexion nor in extension but in rotation. To prevent it, the tenodesis must be fixed in neutral rotation; lateral extraarticular tenodesis is contraindicated in case of postero lateral insufficiency.

Williams: The backlash is about to start! The ALL reconstructions won't work and some techniques will be malplaced and either damage the proximal LCL or restrict flexion. Also there is a theme from lab testing which will show 'over constraint' from ALL recon. Unfortunately, these things may mean that surgeons then move away from these procedures, which may result in abandoning good operations!

Sonnery-Cottet: In our hands, the downsides of ALL reconstruction performed with the current technique are minimal. Moreover, the procedure is compatible with outpatient surgery and the learning curve is relatively short. It is much easier to perform than double bundle.

However, we should note the concerns regarding overconstraint and early degenerative change that have been associated with extra-articular tenodeses such as the Lemaire. These negative reports have been attributed to imperfectly anatomic ACL reconstruction, nonanatomic extra-articular tenodeses and prolonged immobilisation, all of which are not features of modern rehabilitation or anatomic ALL reconstruction.

Musahl: Several studies have shown that overconstraint can be a potential concern. One should also avoid overtensioning of the graft or tensioning the graft at low flexion angles. As described above the ALC is most taut in higher flexion angles. It should also be noted that by definition extra-articular tenodesis procedures, including ALL reconstruction are nonanatomic. Future research will show if posttraumatic OA will be a concern.

Amis: In the past, one complication was herniation of muscle through the ITB donor site, but that should be avoided by the less-invasive methods used now. Another potential downside is that an over-tight graft might over-constrain the rotational laxity of the knee, and that was shown clearly recently by a study which used $88 \mathrm{~N}$ graft tension. Our studies have found that a tenodesis can correct the rotational laxity with only $20 \mathrm{~N}$ tension. That is desirable, because a further potential downside is the possibility of causing lateral compartment degeneration by a combination of excessive contact pressure and alteration of the native articular kinematics; those have been found not to occur with a lower lateral graft/tenodesis tension.

Zaffagnini: The downsides that you can have while you are performing an extra-articular tenodesis is related to the technique that you are using to do that. Indeed, not all the 
procedures are the same because everyone have his own pros and cons. Right now there are different procedure that can create different scenario and different complications in the lateral compartment due to the type of passage and fixation. There are procedures that use a iliotibial tract leaved attached at the Gerdy's and passed below the LCL and fixed on the femur posterior close to the Kaplan fibers. These steps could be responsible of excessive lateral compartment $\mathrm{OA}$ in relation to the overtightening of the lateral compartment with the knee fixed in external rotation especially when this procedure is tightening the external rotation. On the other hand, there are others procedure that starting from the origin of the Kaplan fibers and then goes below the LCL and fixed the graft between the fibular head and the Gerdy's tubercle. This type of procedure avoids the overtightening of the lateral compartment but could have a failure of the graft or at the femur or at the tibial insertion. The last one is the one that we normally use that could use the graft fixed at the femur close to the Kaplan fibers and then below over the fascia below the Gerdy's tubercle. This type of procedure in our experience avoiding the passage below the LCL avoid the risk of overtightening of the lateral compartment. The main drawback is that you have sometimes necessity of removal all the staples.

Karlsson: What do you expect to see in the future of ACL reconstruction surgery relative to recreating the native rotatory stability of the knee?

Amis: Increasing attention to searching for other injuries, such as meniscal-capsular separation or root tears, as well as capsular and extra-capsular peripheral structures, all around the knee. This will be a correction to the intra-articular arthroscopic 'tunnel vision' which has prevailed recently! This will be part of a realisation that an isolated ACL reconstruction cannot fix every aspect of an unstable knee. I also expect the see wider use of instruments to measure rotational laxity.

Zaffagnini: In the future for sure the percentage of extra-articular tenodesis to have better control of rotatory knee instability will increase. For sure adding an extra-articular plasty is not really good and it is not like the normal situation in a native knee. So far, until we are able to define the optimal strategy to restore the native rotational laxity, this type of procedure will proliferate. But the final goal should be to restore the native rotational laxity as anatomic as [possible].

\footnotetext{
Sonnery-Cottet: Unfortunately, whatever the device used, the objective evaluation of knee rotatory instability is a chimera. It can only be objectively evaluated under general anesthesia and not correctly in an awake patient. This considerably decreases its importance, as we do not have an objective measurement before and after the surgery. Instead, surgeons should objectively evaluate their clinical results. We should be honest with our patients and not estimate our outcomes. If you look at the literature, the outcomes are still very disappointing with graft failure rates of 18 to $28 \%$ (mean follow up 2 to 6 years) in a high risk population, re-operation rates of 19 to $26 \%$ and only 50 to $65 \%$ returning to their previous levels of sport. Despite a greater understanding of the anatomy and biomechanics of the ACL, none of the technical innovations in recent history (e.g. double bundle reconstruction or the widespread
} 
change from trans-tibial drilling to anatomic femoral tunnel drilling) have resulted in improved results. However, our retrospective clinical series with combined ACL + ALL reconstruction shows good clinical results without specific complications. A prospective comparative randomized study is in progress to confirm these findings.

Musahl: Large-scale clinical studies will be needed to direct patient care. I also believe that instrumented laxity testing and computer technology will be further developed and will prove useful for the treatment of rotatory knee laxity.

Neyret: I really expect the tools to evaluate the translations and the rotation to be more accurate. With good parameters of assessments, we will improve the understanding of the different anterior chronic laxities. The theory of bumpers developed by Franck Noyes or by Bousquet was probably abandoned by too many surgeons. The key of the treatment for the anterior chronic laxities is probably to address these different abnormal translations and rotations. In others words, the treatment for all the different anterior chronic laxities cannot be compensated by a single ACL reconstruction.

Claes: With regard to the unseen and sometimes harsh controversy the ALL has seen since we have published our anatomical study in 2013[7], I rely on the famous words of the philosopher Arthur Schopenhauer: "All truth passes through three stages. First, it is ridiculed. Second, it is violently opposed. Third, it is accepted as being self-evident."

Williams: The future is about attention to detail and addressing al contributory lesions e.g. meniscal root / ramp lesions, MCL etc. Also we may refine the intra-articular ACL reconstruction (ribbon, etc.)

Getgood: I believe that in the future we will have a much greater understanding of what underpins the existence of variable grades of rotatory laxity. We will have better objective methods available to us in the clinic to assess and measure subtle rotational laxities, and as such, will develop bespoke techniques to address them. Ultimately, as we continue to develop novel techniques, we need more advanced outcome measures so that we can more readily differentiate between surgical procedures to determine improved patient outcomes. Ultimately, improvement in kinematic control is only one piece of the puzzle. This must be combined with a biological approach to address the altered physiology within the joint, not only at the time of injury, but also at the time of reconstruction, when as surgeons we hit the knee with another un-physiological insult. I believe that this tailored 'mechanobiological' approach to ACL injury and reconstruction will ultimately result in the most favorable outcomes for our patients.

\section{Conclusion:}

In summary, some degree of disagreement still exists regarding the role of individual anterolateral structures and the appropriate management of rotatory knee laxity in ACL-deficient knees. However, the overall theme of this consensus is that the anterolateral structures do provide a significant contribution to rotatory knee stability (secondary to the ACL) and should be considered in the setting of ACL injury, especially in cases with a high degree of pivot shift (either clinically or quantitatively) 
646 or in settings of revision surgery. However, it is also crucial to address relevant meniscal lesions, 647 meniscocapsular separations, and risky bony morphology in these patients. Whether discussing the 648 ALC as a whole, or the ALL specifically (including the capsulo-osseous layer of the ITB or the mid649 third capsular ligament), the anterolateral knee structures must be conceptualized not as isolated 650 structures, but rather as key players in a complex team that includes the ACL, the menisci, the 651 capsule, the bony morphology, and other contributing factors to rotatory knee stability. As 652 demonstrated in this round table discussion, all investigators perform lateral tenodesis procedures on a 653 certain percentage of their primary ACL surgeries and an even higher percentage of their revision 654 ACL surgeries. Yet, the best way to manage these injuries is still unknown, although significant 655 progress has been made. In the future, it will be important to perform high level studies with robust 656 outcomes measures to elucidate the appropriate surgical indications and risk factors for these extra657 articular procedures performed concomitantly with ACL reconstruction. 
1. Berruto M, Uboldi F, Gala L, Marelli B, Albisetti W (2013) Is triaxial accelerometer reliable in the evaluation and grading of knee pivot-shift phenomenon? Knee Surg Sports Traumatol Arthrosc 21 (4):981-985.

2. Bignozzi S, Zaffagnini S, Lopomo N, Martelli S, Iacono F, Marcacci M (2009) Does a lateral plasty control coupled translation during antero-posterior stress in single-bundle ACL reconstruction? An in vivo study. Knee Surg Sports Traumatol Arthrosc 17 (1):65-70.

3. Bonanzinga T, Signorelli C, Grassi A, Lopomo N, Bragonzoni L, Zaffagnini S, Marcacci M (2016) Kinematics of ACL and anterolateral ligament. Part I: Combined lesion. Knee Surg Sports Traumatol Arthrosc.

4. Bonanzinga T, Signorelli C, Lopomo N, Grassi A, Neri MP, Filardo G, Zaffagnini S, Marcacci M (2015) Biomechanical effect of posterolateral corner sectioning after ACL injury and reconstruction. Knee Surg Sports Traumatol Arthrosc 23 (10):2918-2924.

5. Caterine S, Litchfield R, Johnson M, Chronik B, Getgood A (2015) A cadaveric study of the anterolateral ligament: re-introducing the lateral capsular ligament. Knee Surg Sports Traumatol Arthrosc 23 (11):3186-3195.

6. Chylarecki C, Hierholzer G, Klose R (1996) [Ultrasound diagnosis of acute rupture of the anterior cruciate ligament. An experimental and clinical study]. Unfallchirurg 99 (1):24-30.

7. Claes S, Vereecke E, Maes M, Victor J, Verdonk P, Bellemans J (2013) Anatomy of the anterolateral ligament of the knee. J Anat 223 (4):321-328.

8. Daggett M, Ockuly AC, Cullen M, Busch K, Lutz C, Imbert P, Sonnery-Cottet B (2016) Femoral Origin of the Anterolateral Ligament: An Anatomic Analysis. Arthroscopy 32 (5):835-841.

9. Early NA, Kelly MJ, Schlosser AK, Filipkowski DE, Elias JJ (2015) Anterolateral ligament injury increases tibiofemoral kinematic changes following Acl injury: A simulation study. Paper presented at the Orthopaedic Research Society, Las Vegas, NV, Mar 28-31

10. Ferretti A, Monaco E, Fabbri M, Maestri B, De Carli A (2016) Prevalence and Classification of Injuries of Anterolateral Complex in Acute Anterior Cruciate Ligament Tears. Arthroscopy.

11. Guenther D, Griffith C, Lesniak B, Lopomo N, Grassi A, Zaffagnini S, Fu FH, Musahl V (2015) Anterolateral rotatory instability of the knee. Knee Surg Sports Traumatol Arthrosc 23 (10):2909-2917.

12. Guenther D, Rahnemai-Azar AA, Bell KM, Irarrazaval S, Fu FH, Musahl V, Debski RE (2016) The Anterolateral Capsule of the Knee Behaves Like a Sheet of Fibrous Tissue. Am J Sports Med.

13. Hughston JC, Andrews JR, Cross MJ, Moschi A (1976) Classification of knee ligament instabilities. Part II. The lateral compartment. J Bone Joint Surg Am 58 (2):173-179.

14. Hughston JC, Jacobson KE (1985) Chronic posterolateral rotatory instability of the knee. J Bone Joint Surg Am 67 (3):351-359.

15. Journées Lyonnaises de Chirurgie du Genou (2002). Lyon

16. Kaplan EB (1958) The iliotibial tract; clinical and morphological significance. J Bone Joint Surg Am 40-A (4):817-832.

17. Kennedy MI, Claes S, Fuso FA, Williams BT, Goldsmith MT, Turnbull TL, Wijdicks CA, LaPrade RF (2015) The Anterolateral Ligament: An Anatomic, Radiographic, and Biomechanical Analysis. Am J Sports Med 43 (7):1606-1615.

18. Kittl C, El-Daou H, Athwal KK, Gupte CM, Weiler A, Williams A, Amis AA (2016) The Role of the Anterolateral Structures and the ACL in Controlling Laxity of the Intact and ACL-Deficient Knee. Am J Sports Med 44 (2):345-354.

19. LaPrade RF (2016) Editorial Commentary: Defining the Anatomy of the Anterolateral Aspect of the Knee Among Experts Is Clearly Needed. Arthroscopy 32 (5):842-843.

20. Marcacci M, Zaffagnini S, Giordano G, Iacono F, Presti ML (2009) Anterior cruciate ligament reconstruction associated with extra-articular tenodesis: A prospective clinical and radiographic evaluation with 10- to 13-year follow-up. Am J Sports Med 37 (4):707-714.

21. Marcacci M, Zaffagnini S, Iacono F, Neri MP, Loreti I, Petitto A (1998) Arthroscopic intra- and extraarticular anterior cruciate ligament reconstruction with gracilis and semitendinosus tendons. Knee surgery, sports traumatology, arthroscopy : official journal of the ESSKA 6 (2):68-75.

22. Monaco E, Ferretti A, Labianca L, Maestri B, Speranza A, Kelly MJ, D'Arrigo C (2012) Navigated knee kinematics after cutting of the ACL and its secondary restraint. Knee Surg Sports Traumatol Arthrosc $20(5): 870-877$.

23. Muller W (1977) [Current aspects of functional anatomy of the knee joint]. Hefte Unfallheilkd (129):131137.

24. Musahl V, Citak M, O'Loughlin PF, Choi D, Bedi A, Pearle AD (2010) The effect of medial versus lateral meniscectomy on the stability of the anterior cruciate ligament-deficient knee. Am J Sports Med 38 (8):1591-1597. 
J, Group PS (2016) Validation of Quantitative Measures of Rotatory Knee Laxity. Am J Sports Med 44 (9):2393-2398.

26. Musahl V, Rahnemai-Azar AA, Costello J, Arner JW, Fu FH, Hoshino Y, Lopomo N, Samuelsson K, Irrgang JJ (2016) The Influence of Meniscal and Anterolateral Capsular Injury on Knee Laxity in Patients With Anterior Cruciate Ligament Injuries. Am J Sports Med 44 (12):3126-3131.

27. Musahl V, Rahnemai-Azar AA, van Eck CF, Guenther D, Fu FH (2016) Anterolateral ligament of the knee, fact or fiction? Knee Surg Sports Traumatol Arthrosc 24 (1):2-3.

28. Nakamura N, Rodeo SA, Alini M, Maher S, Madry H, Erggelet C (2015) Physiology and Pathophysiology of Musculoskeletal Tissues. In: Miller M, Thompson S (eds) DeLee \& Drez's Orthopaedic Sports Medicine: Principles and Practice. 4th edn.,

29. Nitri M, Rasmussen MT, Williams BT, Moulton SG, Cruz RS, Dornan GJ, Goldsmith MT, LaPrade RF (2016) An In Vitro Robotic Assessment of the Anterolateral Ligament, Part 2: Anterolateral Ligament Reconstruction Combined With Anterior Cruciate Ligament Reconstruction. Am J Sports Med 44 (3):593-601.

30. Parsons EM, Gee AO, Spiekerman C, Cavanagh PR (2015) The biomechanical function of the anterolateral ligament of the knee. Am J Sports Med 43 (3):669-674.

31. Rahnemai-Azar AA, Abebe ES, Johnson P, Labrum J, Fu FH, Irrgang JJ, Samuelsson K, Musahl V (2016) Increased lateral tibial slope predicts high-grade rotatory knee laxity pre-operatively in ACL reconstruction. Knee Surg Sports Traumatol Arthrosc.

32. Rahnemai-Azar AA, Miller RM, Guenther D, Fu FH, Lesniak BP, Musahl V, Debski RE (2016) Structural Properties of the Anterolateral Capsule and Iliotibial Band of the Knee. Am J Sports Med 44 (4):892897.

33. Rasmussen MT, Nitri M, Williams BT, Moulton SG, Cruz RS, Dornan GJ, Goldsmith MT, LaPrade RF (2016) An In Vitro Robotic Assessment of the Anterolateral Ligament, Part 1: Secondary Role of the Anterolateral Ligament in the Setting of an Anterior Cruciate Ligament Injury. Am J Sports Med 44 (3):585-592.

34. Rezansoff AJ, Caterine S, Spencer L, Tran MN, Litchfield RB, Getgood AM (2015) Radiographic landmarks for surgical reconstruction of the anterolateral ligament of the knee. Knee Surg Sports Traumatol Arthrosc 23 (11):3196-3201.

35. Runer A, Birkmaier S, Pamminger M, Reider S, Herbst E, Kunzel KH, Brenner E, Fink C (2016) The anterolateral ligament of the knee: A dissection study. Knee 23 (1):8-12.

36. Schon JM, Moatshe G, Brady AW, Serra Cruz R, Chahla J, Dornan GJ, Turnbull TL, Engebretsen L, LaPrade RF (2016) Anatomic Anterolateral Ligament Reconstruction of the Knee Leads to Overconstraint at Any Fixation Angle. Am J Sports Med 44 (10):2546-2556.

37. Signorelli C, Bonanzinga T, Lopomo N, Marcheggiani Muccioli GM, Bignozzi S, Filardo G, Zaffagnini S, Marcacci M (2013) Do pre-operative knee laxity values influence post-operative ones after anterior cruciate ligament reconstruction? Scand J Med Sci Sports 23 (4):e219-224.

38. Signorelli C, Filardo G, Bonanzinga T, Grassi A, Zaffagnini S, Marcacci M (2016) ACL rupture and joint laxity progression: a quantitative in vivo analysis. Knee Surg Sports Traumatol Arthrosc 24 (11):36053611 .

39. Song GY, Zhang H, Wang QQ, Zhang J, Li Y, Feng H (2016) Risk Factors Associated With Grade 3 Pivot Shift After Acute Anterior Cruciate Ligament Injuries. Am J Sports Med 44 (2):362-369.

40. Sonnery-Cottet B, Archbold P, Zayni R, Bortolletto J, Thaunat M, Prost T, Padua VB, Chambat P (2011) Prevalence of septic arthritis after anterior cruciate ligament reconstruction among professional athletes. Am J Sports Med 39 (11):2371-2376.

41. Sonnery-Cottet B, Thaunat M, Freychet B, Pupim BH, Murphy CG, Claes S (2015) Outcome of a Combined Anterior Cruciate Ligament and Anterolateral Ligament Reconstruction Technique With a Minimum 2Year Follow-up. Am J Sports Med 43 (7):1598-1605.

42. Stephen JM, Halewood C, Kittl C, Bollen SR, Williams A, Amis AA (2016) Posteromedial Meniscocapsular Lesions Increase Tibiofemoral Joint Laxity With Anterior Cruciate Ligament Deficiency, and Their Repair Reduces Laxity. Am J Sports Med 44 (2):400-408.

43. Tavlo M, Eljaja S, Jensen JT, Siersma VD, Krogsgaard MR (2016) The role of the anterolateral ligament in ACL insufficient and reconstructed knees on rotatory stability: A biomechanical study on human cadavers. Scand J Med Sci Sports 26 (8):960-966.

44. Terry GC, Hughston JC, Norwood LA (1986) The anatomy of the iliopatellar band and iliotibial tract. Am J Sports Med 14 (1):39-45.

45. Thein R, Boorman-Padgett J, Stone K, Wickiewicz TL, Imhauser CW, Pearle AD (2016) Biomechanical Assessment of the Anterolateral Ligament of the Knee: A Secondary Restraint in Simulated Tests of the Pivot Shift and of Anterior Stability. J Bone Joint Surg Am 98 (11):937-943. 
46. Trojani C, Beaufils P, Burdin G, Bussiere C, Chassaing V, Djian P, Dubrana F, Ehkirch FP, Franceschi JP, Hulet C, Jouve F, Potel JF, Sbihi A, Neyret P, Colombet P (2012) Revision ACL reconstruction: influence of a lateral tenodesis. Knee Surg Sports Traumatol Arthrosc 20 (8):1565-1570.

47. Van der Watt L, Khan M, Rothrauff BB, Ayeni OR, Musahl V, Getgood A, Peterson D (2015) The structure and function of the anterolateral ligament of the knee: a systematic review. Arthroscopy 31 (3):569-582 e563.

48. Van Dyck P, Clockaerts S, Vanhoenacker FM, Lambrecht V, Wouters K, De Smet E, Gielen JL, Parizel PM (2016) Anterolateral ligament abnormalities in patients with acute anterior cruciate ligament rupture are associated with lateral meniscal and osseous injuries. Eur Radiol 26 (10):3383-3391.

49. Vincent JP, Magnussen RA, Gezmez F, Uguen A, Jacobi M, Weppe F, Al-Saati MF, Lustig S, Demey G, Servien E, Neyret P (2012) The anterolateral ligament of the human knee: an anatomic and histologic study. Knee Surg Sports Traumatol Arthrosc 20 (1):147-152.

50. Zantop T, Schumacher T, Diermann N, Schanz S, Raschke MJ, Petersen W (2007) Anterolateral rotational knee instability: role of posterolateral structures. Winner of the AGA-DonJoy Award 2006. Arch Orthop Trauma Surg 127 (9):743-752.

51. Zens M, Niemeyer P, Ruhhammer J, Bernstein A, Woias P, Mayr HO, Sudkamp NP, Feucht MJ (2015) Length Changes of the Anterolateral Ligament During Passive Knee Motion: A Human Cadaveric Study. Am J Sports Med 43 (10):2545-2552. 


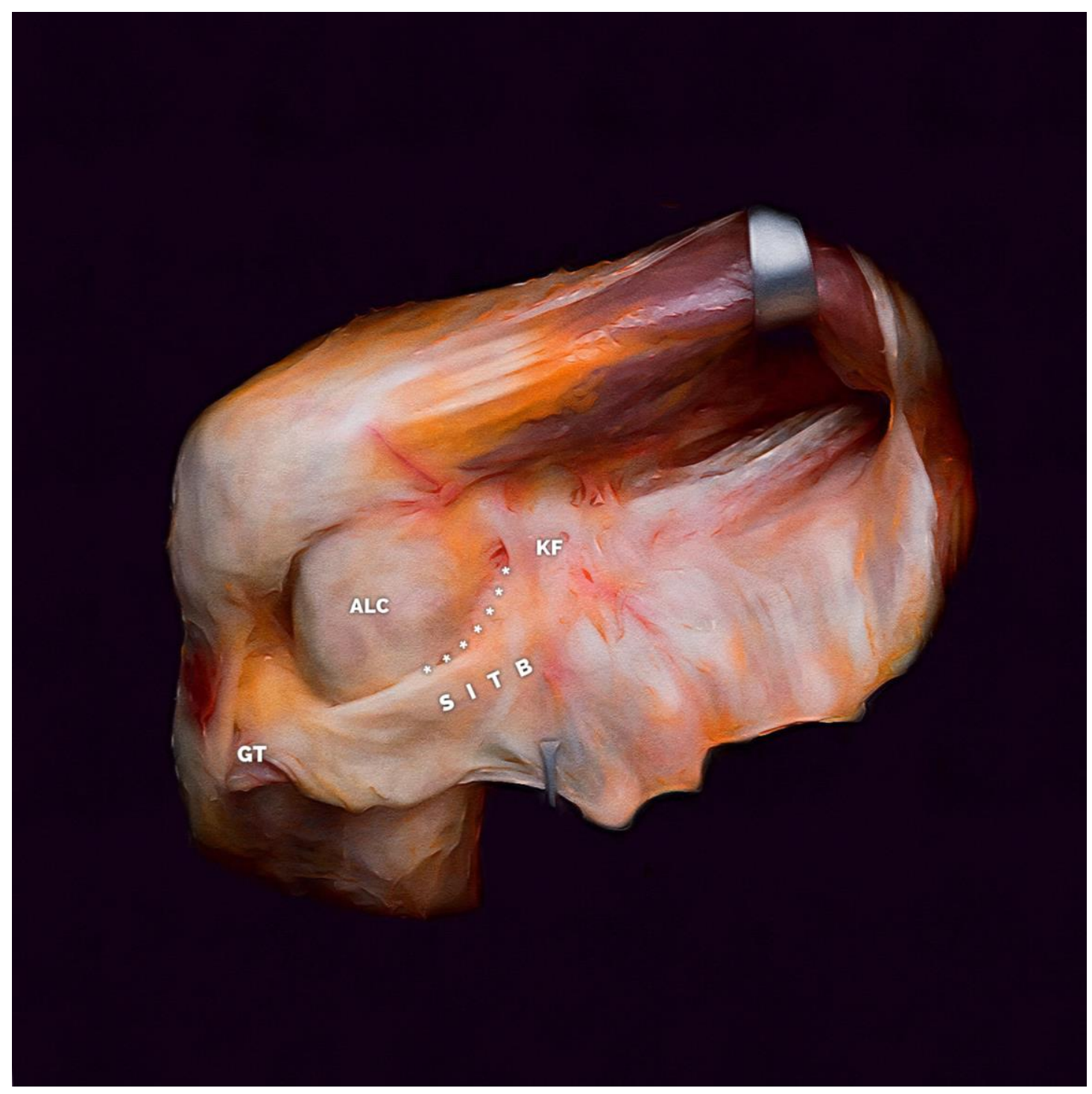

Figure 1: The Anterolateral Complex. View of a layer-by-layer dissection of the left knee, demonstrating the key structures of the anterolateral complex. The superficial iliotibial band (SITB) inserts on a wide area of the proximal tibia that includes Gerdy's tubercle (GT) anteriorly, as well as the anterolateral and lateral proximal tibia. The Kaplan fibers (KF) connect the SITB to the distal femoral metaphysis and lateral condyle. The anterolateral capsule (ALC) contains superficial and deep layers, with the lateral collateral ligament (not pictured) located between the two layers. The two capsular layers merge into one layer anteriorly. The capsuloosseous layer of the ITB (denoted by *) is continuous with the lateral gastrocnemius muscle fascia and and the lateral femoral epicondyle proximally, and then merges with the ITB distally and inserts midway between the fibular head and GT. Some authors have suggested that the structure commonly described as the anterolateral ligament could be the capsulo-osseous layer of the ITB or the confluence of the superficial and deep layers of 


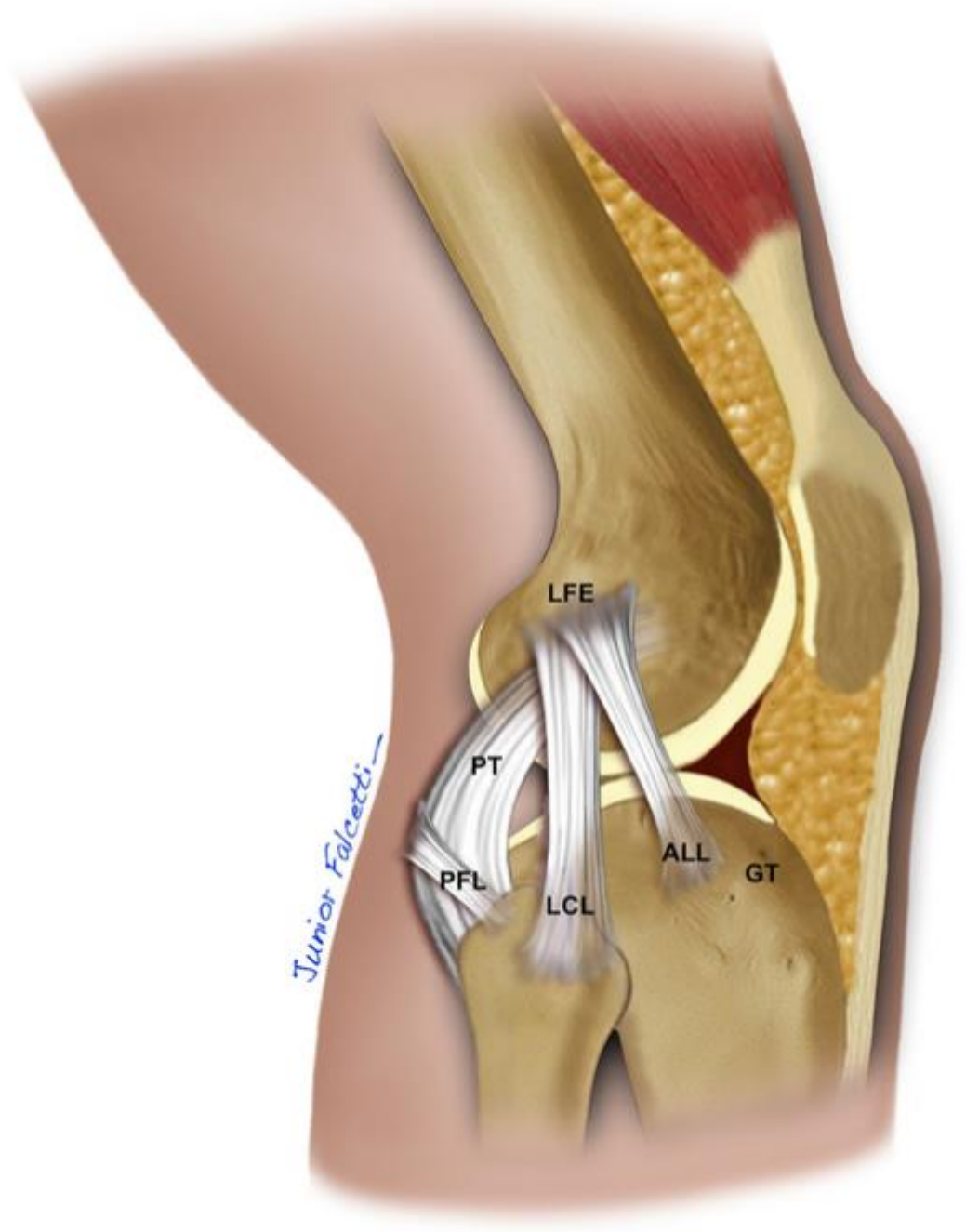

Figure 2: The Anterolateral Ligament. Artistic rendering of the anterolateral ligament (ALL) of the right knee, as described in previous studies. $L F E$ lateral femoral epicondyle, $A L L$ anterolateral ligament, $G T$ Gerdy's tubercle, $P T$ popliteus tendon, $P F L$ popliteal fibular ligament.

Reproduced from: Ingham, S.J.M., de Carvalho, R.T., Martins, C.A.Q. et al. Knee Surg Sports Traumatol Arthrosc (2015). doi:10.1007/s00167-015-3956-2 